\title{
ENVIRONMENTAL RESEARCH
}

LETTERS

LETTER • OPEN ACCESS

Saving the world from your couch: the heterogeneous medium-run benefits of COVID-19 lockdowns on air pollution

To cite this article: Jean-Philippe Bonardi et al 2021 Environ. Res. Lett. 16074010

View the article online for updates and enhancements. 


\title{
ENVIRONMENTAL RESEARCH
}

\section{LETTERS}

\section{LETTER}

CrossMark

OPEN ACCESS

RECEIVED

7 December 2020

REVISED

3 March 2021

ACCEPTED FOR PUBLICATION

12 March 2021

PUBLISHED

25 June 2021

Original Content from

this work may be used

under the terms of the

Creative Commons

Attribution 4.0 licence.

Any further distribution

of this work must

maintain attribution to

the author(s) and the title

of the work, journal

citation and DOI.

\section{Saving the world from your couch: the heterogeneous medium-run benefits of COVID-19 lockdowns on air pollution}

\author{
Jean-Philippe Bonardi ${ }^{1,4, *}$, Quentin Gallea ${ }^{2,4}$, Dimitrija Kalanoski ${ }^{3,4}$, Rafael Lalive $^{1,4}\left(\mathbb{C}\right.$, Raahil Madhok $^{5}$, \\ Frederik Noack $^{5}$ (D), Dominic Rohner ${ }^{1,4}$ (i) and Tommaso Sonno ${ }^{6,7}$ (i) \\ 1 Faculty of Business and Economics (HEC Lausanne), University of Lausanne, Lausanne, Switzerland \\ 2 Department of Economics, University of Zurich, Zurich, Switzerland \\ 3 Alliance Manchester Business School, University of Manchester, Manchester, United Kingdom \\ 4 Enterprise for Society (E4S) Center, Lausanne, Switzerland \\ 5 Department of Food and Resource Economics, University of British Columbia, Vancouver, Canada \\ 6 Department of Economics, University of Bologna, Bologna, Italy \\ 7 Centre for Economic Performance, London School of Economics and Political Science, London, United Kingdom \\ * Author to whom any correspondence should be addressed. \\ E-mail: Jean-Philippe.Bonardi@unil.ch
}

Keywords: COVID-19, pollution, lockdown, sustainable development, environment, globalization

\begin{abstract}
In Spring 2020, COVID-19 led to an unprecedented halt in public and economic life across the globe. In an otherwise tragic time, this provides a unique natural experiment to investigate the environmental impact of such a (temporary) 'de-globalization'. Here, we estimate the medium-run impact of a battery of COVID-19 related lockdown measures on air quality across 162 countries, going beyond the existing short-run estimates from a limited number of countries. In doing so, we leverage a new dataset categorizing lockdown measures and tracking their implementation and release, extending to 31 August 2020. We find that domestic and international lockdown measures overall led to a decline in PM2.5 pollution by $45 \%$ and 35\%, respectively. This substantial impact persists in the medium-run, even as lockdowns are lifted, there is, however, substantial heterogeneity across different types of lockdown measures, different countries, and different sources of pollution. We show that some country trajectories are much more appealing (with fewer COVID-19 casualties, less economic downturn and bigger pollution reductions) than others. Our results have important policy implications and highlight the potential to 'build back better' a sustainable economy where pollution can be curbed in a less economically costly way than during the COVID-19 pandemic.
\end{abstract}

\section{Introduction}

Major pandemics do not only bring great harm and suffering to humanity, but also allow for opportunities in innovation and to 'build back better'. If the plague spurred on a series of medical and organizational innovations, such as quarantines and modern public administrations, then the current COVID-19 pandemic has championed remote working, various dimensions of digitalization, and virtual meetings. Altered patterns of mobility, work organization, and slow-downs in production could be cornerstones for more wide-ranging societal changes and new approaches to saving the environment.
Air pollution is among the most severe environmental problems. It causes several million deaths every year, disproportionately affecting the global poor [26]. Pollution is also directly related to economic activity and air quality is therefore likely to improve with economic restrictions, which have been the primary response to the COVID-19 pandemic. Such restrictions, termed non-pharmaceutical interventions (NPIs), range in stringency from school closures to full economic shutdowns and curfews. Throughout this paper, we use the term 'lockdown' and 'NPI' interchangeably, and specify when referring to a specific type of measure. Although the relationship between economic activity and air pollution is 
thought to be generally positive, the strength of this relationship could vary across the globe in intricate ways. Heterogeneity in the response to air pollution across regions, or lockdown measures that target specific economic activities, can therefore inform us on possibilities in improving the environment without sacrificing economic prosperity.

In order to assess how various facets of COVIDinduced lockdowns have impacted air pollution in the short- and medium-run, one needs a global study of the impact of a whole arsenal of lockdown measures for the entire globe, and covering the period from the COVID-19 pandemic beginning until today.

While there exist pioneering estimates of the lockdowns' impact on environmental outcomes, most studies focus on a single country $[1,5,10,12,14,27$, $33,39,42,44]$, or lump together the whole range of NPIs into one aggregate variable [15, 17, 28, 43]. Only one paper [31] studies the impact of eight types of lockdown measures on pollution levels, covering the time period from the 1 January 2020 to the 5 July 2020 for 76 countries.

Contrary to existing articles, here we focus not just on short-run but also on medium-run consequences of lockdown measures. This longer-run impact assessment allows us to evaluate the sustainability of particular policies for bringing the environment 'back on track'. Medium-run evidence provides precious information on the extent of air quality improvements that are attainable-key information for 'building back better' our societies and economies post-COVID-19.

More precisely, by building on existing work, we extend the framework of analysis in several dimensions. Our data covers a time period that is several months longer than those of existing papers, which allows us to move beyond short-run effects and assess whether potential environmental benefits extend to the medium run. In doing so, we explore the question of whether lockdown releases in many countries over the summer 2020 [2] have reversed previous environmental gains, or if they persist even after lockdowns were lifted.

The first key data source to assess the pollution impact of specific lockdown measures is a dataset covering a global sample of 162 countries, and distinguishing several types of measures ranging from partial to full lockdowns and from within-country (hereafter, 'inside') measures, such as school closures and curfews, to international (hereafter, 'outside') measures, such as national border closures. Such detailed information on lockdown measures allows us to discuss whether and how the strictness of a policy matters to its air quality impact. Starting from a new and fine-grained dataset on lockdown measures [3], we extend the time horizon of this data, and add information on lockdown releases. This makes ours the first paper that studies not only the impact of COVID-19 related lockdowns on air pollution, but also of lockdown releases.

To generate the data, we relied on a customcoded JAVA web scraping program that extracted all news headlines per country from 31 October 2019 to 31 August 2020. From this, we coded the lockdown/lockdown release measures. These were further cross-checked with COVID-19 announcements from the US embassy COVID-19 bulletin, which provides news coverage for all countries. Our lockdown/lockdown release dataset: (i) allows precise identification of the governmental measures that significantly hamper the movement of individuals, (ii) identifies the earliest date when a measure was activated (for lockdowns) or deactivated (for lockdown release), and (iii) differentiates between inside and outside lockdown/lockdown release measures that impacted the movement of individuals ${ }^{8}$. Further details on this data are provided below in section 3 Methods.

The second key data for our analysis is data on pollution. While pollution monitoring networks exist worldwide, they only offer sparse geographic coverage. We overcome this by taking advantage of daily, high-resolution satellite-based pollutant retrievals from NASA [18]. The data product assimilates satellite-measured aerosols into a gridded $\left(0.5^{\circ} \times\right.$ $\left.0.625^{\circ}\right)$, high-frequency dataset with complete global coverage. We restrict our PM2.5 $\left(\mu \mathrm{g} \mathrm{nm}^{-3}\right)$ measure to urban areas, where lockdowns are expected to starkly reduce pollution-generating human activity.

A key concern in the environmental economic literature is the spatial correlation of economic activity and pollution. With regards to COVID-19, the timing of economic lockdowns is arguably correlated between nearby countries, and pollution in one country will be affected by the lockdown of its neighbour. Previous studies on COVID-19 and air quality have not accounted for such indirect channels. Here, we advance the literature by tracking corresponding NPI timelines for all adjacent countries and separate out the indirect effect in our analysis. As such, in addition to accounting for lockdown release, we are also the first to disentangle spatial spillovers.

For our pollution indicator, we focus on PM2.5 because it stands out as the pollutant with the most acute mortality consequences. Indeed, the World Health Organization uses PM2.5 as its main indicator of population exposure to pollution. Furthermore, we opt for remotely sensed data over ground monitor

\footnotetext{
8 This differs from alternative (and complementary) approaches such as the one followed by [24] that considers a broad range of NPIs, including some that are less related to mobility patterns. Given that our research question focuses on mobility and pollution, our data is more suitable for the precise purpose of the current study.
} 
data-which has been used in previous global studies of lockdown impacts [31, 43]-for two reasons. First, the number and location of monitors varies widely by country, and are often endogenously placed avoiding areas of high pollution [19]. In contrast, our gridded data is more representative-equivalent to placing a monitor roughly every $50 \mathrm{~km}$ worldwide. Second, pre-publication QA/QC protocols vary widely and efforts to synthesize monitor data provide little guidance on data flagging, while the atmospheric model underlying our data ensures a unified pollution measure based on consistent physical and chemical transport properties. Further details on the pollution data used are provided in the next section.

\section{Data}

\subsection{Lockdown data}

The lockdown data from [3] assembled information on each country's lockdown policies, relying on web-scraping, and drawing on news headlines published between 31 October 2019 to 15 October 2020, provided by LexisNexis. They crosschecked this data with country information from COVID-19 bulletins issued by the United States Embassy. Their final dataset contains the dates of implementation and release for a series of specific NPIs designed to stop the spread of the COVID-19. The following categories of NPI are distinguished: within country regional lockdown, partial selective lockdown (prohibiting some activities), curfew and state of emergency, country national lockdown, selective border closure stage 1 and 2, and country international lockdown. This dataset was extended here, with similar methods, to include the lockdown release period.

Furthermore, we also control a series of sanitary indicators. Specifically, data on COVID-19 fatalities stems from the Johns Hopkins University [16], which is arguably the most complete and reliable source available.

The final dataset covers 162 countries over the time spanning from the 1 November 2019 to the 31 August 2020.

\subsection{Pollution data}

Significant strides have recently been made to increase the quality and scope of satellite pollution data. For example, the MODIS instrument aboard NASA's Aqua and Terra spacecrafts measures high resolution aerosol optical depth (AOD). AOD measures sunlight reflected by suspended particulates and is often used to proxy PM2.5 ${ }^{9}[21,30,34]$. However, cloud contamination and sensor-specific data gaps lower data quality. Recognizing this, [45] develop a method for isolating fAOD-a specific component of AOD known for its importance in maintaining

\footnotetext{
9 This equivalence is limited because AOD is an atmospheric measure whereas PM2.5 is a surface measurement.
}

global water, carbon and nitrogen cycles-and validate it against ground monitors from several countries ${ }^{10}$. Lastly, the Modern Era Retrospective Analysis for Research and Applications (MERRA-2) [18] data product offers another advancement by assimilating raw AOD retrievals through a global atmospheric circulation model to provide a unified set of groundlevel estimates of five particulate species.

Acknowledging the benefits offered by each data system, here we opt to use MERRA-2 because of its convenience for deriving PM2.5 concentrations. We are specifically interested in PM2.5 because of its global importance as an indicator of human health and because it is largely made up of anthropogenic sources. Furthermore, MERRA-2 offers complete global coverage allowing us to study pollution trends where ground monitor data is unavailable or unreliable.

To achieve global coverage, MERRA-2 combines satellite-measured aerosol and meteorological variables with the GEOS-5 chemical transport model [38] to produce ground-level particulate estimates. GEOS-5 is a detailed climate model that includes atmospheric circulation, oceanic, and land components. Importantly, it incorporates aerosol processes from the Goddard Chemistry, Aerosol, Radiation, and Transport (GOCART) model which assimilates bias-corrected AOD retrievals from MODIS [11]. The algorithm uses a neural net to fill data gaps from cloud contamination and translate cloud-cleared MODIS reflectances into ground-monitor calibrated aerosol levels.

The GOCART module of GEOS-5 simulates five types of aerosols which we use to estimate PM2.5: dust, sea salt (SS), black carbon (BC), organic carbon (OC), and sulfate $\left(\mathrm{SO}_{4}\right)$. We follow [7] and calculate PM2.5 as:

$$
\begin{aligned}
\text { PM2.5 }= & {\left[\text { DUST }_{2.5}\right]+\left[\mathrm{SS}_{2.5}\right]+[\mathrm{BC}] } \\
& +1.4[\mathrm{OC}]+1.375\left[\mathrm{SO}_{4}\right] .
\end{aligned}
$$

This method is useful insofar as there is a high correlation between derived PM2.5 and actual ground concentrations. Although no single study has globally validated equation (1), several case studies across the developed and developing world show that it matches well with ground concentrations. Notably, [7] validate equation (1) against 150 ground monitors in the U.S. between 2003 and 2012 and find a correlation coefficient of 0.8. Provençal et al [37] do the same across most of Europe using a vast network of monitors and find that derived PM2.5 is well simulated. Equation (1) has also been validated in China, Israel, and Taiwan $[23,36]$. The correlation is especially strong in Israel, a region with significant PM2.5

\footnotetext{
10 The global fAOD data developed by [45] spans 2008-2017 and does not overlap with our study period. We are therefore unable to validate our results against this dataset.
} 
loading from desert dust, suggesting derived PM2.5 can be reliably extended to nearby Saharan countries with similar PM2.5 signatures. The correlation coefficient is 0.7 in some parts of China, a region regularly exposed to high-pollution episodes (e.g. crop fires) and with similar PM2.5 sources as nearby South Asia. Lastly, [6] show that MERRA-2 can also pick up major aerosol events, including Saharan dust storms and California wildfires. This is important because COVID-19 lockdowns can also be categorized as a major pollution-relevant event. Taken together, we are confident our derived measure reliably captures global PM2.5 and accurately depicts ground-level concentrations relevant for human health.

We collect MERRA-2 data from the M2T1NXAER files distributed by NASA ${ }^{11}$. These provide hourly aerosol concentrations on a global grid at $0.5^{\circ} \times$ $0.625^{\circ}$ resolution. We produce a daily pollution panel in three steps. First, we aggregate hourly data to the daily mean in each grid cell for the period 1 November 2019-31 August 2020. Second, we extract pollution over urban areas using shapefiles of urban extent as identified by [41]. There are 11878 urban areas, and we extract daily mean pollution over each area for our study period. Lastly, we identify the country of each urban area using a digital world map from www.naturalearthdata.com, and aggregate daily average pollution from urban centres in each country. Our final pollution dataset is a country-day panel of mean PM2.5 in urban areas.

The data on the main source of air pollution are from the Extended Data figure 1 of [29].

\subsection{GDP and COVID-19 mortality data}

Quarterly real GDP data are from the OECD reported by individual countries according to the 2008 System of National Accounts ${ }^{12}$. The data on the number of Covid deaths are from the European Centre for Disease Prevention and Control accessed through the 'our World in Data' website. We restrict the number of deaths to the number of death in the second quarter of 2020 .

\subsection{Meteorological covariates}

A key empirical concern is that the cascade of global COVID-19 lockdowns is correlated with contemporaneous changes in atmospheric conditions that also affect PM2.5 levels. To isolate the lockdown channel, we collect data on a range of time-varying meteorological variables and control for them in the analysis.

We obtain daily satellite data for weather (rainfall and temperature), surface wind speed, humidity, and the planetary boundary layer (PBL) during our study period. Controlling for temperature is important because high temperatures increase photochemical reactions among precursors of PM2.5. Changing

\footnotetext{
11 Retrieved from: https://disc.gsfc.nasa.gov/.

12 Retrieved from: https://data.oecd.org/gdp/quarterly-gdp.htm.
}

wind speed and humidity affects the diffusion of dust particles that make up PM2.5 $[9,13]$. Lastly, the PBL, which is the lowest portion of the troposphere, regulates the upward dispersion of pollutants and has been shown to correlate with PM2.5 formation [32].

Data on rainfall is from the NASA-operated GPM Level-3 product, which provides daily precipitation estimates $($ in $\mathrm{mm}$ ) on a $0.1 \times 0.1$ degree grid [25] All other variables are obtained from the MERRA2 M2T1NXFLX data files, which are provided at the same resolution as the pollution data. Surface wind speed is measured in $\mathrm{m} \mathrm{s}^{-1}$, temperature is measured in Kelvin, humidity is measured as the fraction of water vapour in dry air, and PBL is measured in metres. All covariates are assembled into a countryday panel over urban centres following the same procedure described in Section 2.2.

\section{Methods}

\subsection{Estimation strategy}

Studying the impact of lockdown measures on air quality is statistically challenging, because of related variables affecting both (e.g. a populist government both opposed to certain sanitary measures and in favour of relaxing environmental protection). Seasonal changes in pollution-such as lower air quality in warmer temperatures-also make it difficult to separate the lockdown effect from the onset of summer in the Northern Hemisphere. Lastly, spatial spillovers are pervasive and have previously been unaddressed in the literature. For example, Singapore entered lockdown earlier than neighbouring Malaysia, possibly causing improved air quality in Malaysia despite it not going into total lockdown.

We address endogeneity, omitted variable bias, spillovers, and measurement errors with a large battery of controls and fixed effects. Notably, our finegrained panel data enables the use of country-month fixed effects, which account for unobserved, country specific factors common across all days of the month. The geographic dimension of this demanding specification accounts for the confounding impacts of: geography, industry shares, pollution regulations, population size, topographic features, and any other time-invariant geographic feature entangling the lockdown-pollution relationship. The separate intercept by month removes time-varying biases accruing over wider time scales across the country, such as: the evolution of public perception of the virus, response to global announcements, and changes to the healthcare system.

Beyond fixed effects, we also control for a range of meteorological determinants of PM2.5 formation that change during the same period as lockdown: rainfall $(\mathrm{mm})$, humidity $(\%)$, temperature $(\mathrm{K})$, wind speed $\left(\mathrm{m} \mathrm{s}^{-1}\right)$ and planetary boundary 
layer height $(\mathrm{PBLH})(\mathrm{m})$. See the section titled Meteorological covariates for more details. Crucially, we also account for spatial correlation by controlling for the proportion of bordering countries having implemented an NPI in each time period. We do this to separate the impact of a country's lockdown on its own pollution from the indirect impact from the lockdown of its neighbours. Lastly, we control for the number of deaths within the country, a metric widely accessible to the population, which might drive the preventative behaviour of citizens and firms even in the absence of strict NPI measures.

A remaining concern is that our study period, which extends to the medium term (90-120 days), includes the period when many countries had lifted their respective lockdowns. This generates an offsetting increase in pollution that may negate initial air quality gains, leaving the net effect ambiguous. To guard against this, we use our custom scraped release data to separate out the impact of lockdown from lockdown release.

\subsection{Event study specification}

To study the impact of COVID-19 lockdown measures on global air quality, we implement the following panel OLS regression:

$$
\begin{aligned}
\log \left(P M 2.5_{i t}\right)= & \beta_{k} \sum_{k=-60}^{120} 1\left[t-N P I_{i j}=k\right] \\
& +\beta_{1}\left[\text { Release }_{i j t}\right]+\beta_{2}\left[N b r_{i t}\right] \\
& +\beta_{3}\left[\log \left(\text { Deat }_{i t}+1\right)\right]+\beta_{4}\left[M_{i t}\right] \\
& +\gamma_{i m}+\epsilon_{i t}
\end{aligned}
$$

where $\log \left(P M 2.5_{i t}\right)$ is the $\log$ of PM2.5, normalized by standard deviations, in country $i$ at date $t$. $N P I_{i j}$ is the date of lockdown of type $j$ (e.g. inside measures, outside measures, state of emergency, etc), and $1\left[t-N P I_{i j}=k\right]$ is a dummy that switches on $k$ periods before or after the measure is implemented. The period $k=-1$ is omitted so that all coefficients $\beta_{k}$ are relative to the day before the lockdown event. Release $e_{i j t}$ is a dummy equal to one from the day country $i$ released measure $j . N b r_{i t}$ is the proportion of countries bordering $i$ having implemented any NPI at time $t$, and controls for spatial spillovers. $\log \left(\right.$ Deat $\left._{i t}+1\right)$ captures the timeline of COVID-19 deaths and controls for the evolution of the virus within country $i . M_{i t}$ is a vector of meteorological controls including rainfall, temperature, wind speed, humidity, and PBLH. $\gamma_{i m}$ are country-month fixed effects and $\epsilon_{i t}$ is the error term, clustered at the country level.

Our coefficients of interest are the set of $\beta_{k}$ 's. These represent the global average percentage change in PM2.5 at $k$ days before or after lockdown, relative to the day before lockdown, after controlling for covariates and fixed effects. Importantly, the regression

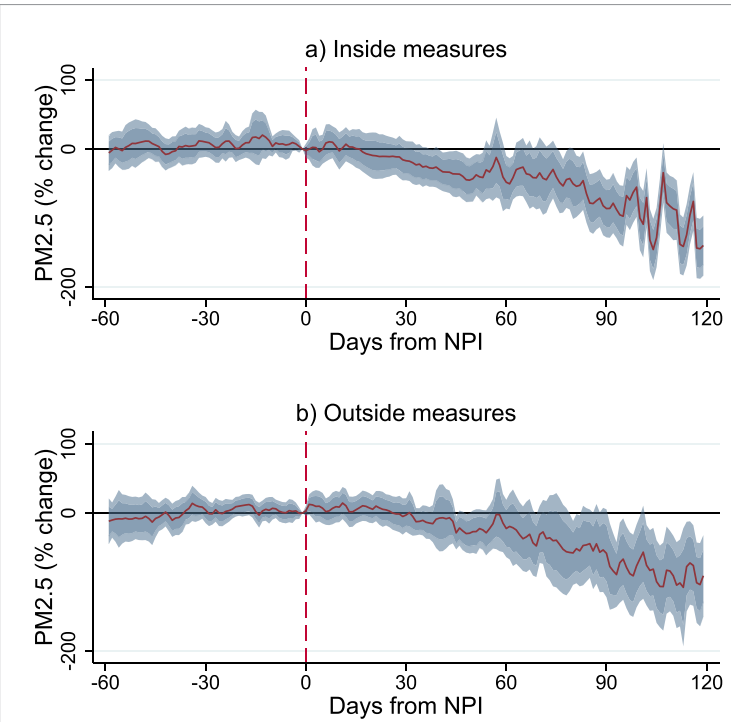

Figure 1. Lockdown measures reduced PM2.5 on the medium term similarly for inside measures (within country) and measures towards the outside (blocking borders). $90 \%$ and $99 \%$ confidence intervals are shown in different shades of blue. The vertical dashed line shows the day when the measure was implemented.

is weighted by the number of urban regions in each country. We do this because, in some countries with few urban areas, our pollution measure is a mean over one or two places (less precise) compared with larger countries where pollution is a mean over hundreds of cities (more precise). Weighting the regression by number of urban areas gives each observation influence over coefficients in proportion to its measurement precision rather than be treated equally.

\section{Results}

Figure 1 presents our main results. It depicts the short-run (1-60 days) and medium-run (61-120 days) global impact of COVID-19 lockdowns on air quality. Two categories of measures are distinguished: Panel (a) reports findings for inside measures, while panel (b) displays the results for outside measures. For each country, we control for the varied timing of lockdown release as well as the proportion of bordering countries having implemented any type of NPI on a given day. Our results can thus be interpreted as a net effect after adjusting for the offsetting effect of lifting lockdowns and removing spatial spillovers.

There are three noteworthy results. First, both inside (panel a) and outside mobility restrictions (panel b) improve air quality over the short and medium run. Over the full period, on average, inside and outside lockdowns reduce PM2.5 concentrations by $45 \%$ and $35 \%$, respectively, relative to the day before its implementation ${ }^{13}$. Second, in the absence

\footnotetext{
13 These results refer to the mean of daily coefficients in figure 1 post-lockdown and are intended only to summarize the event study into a single result.
} 

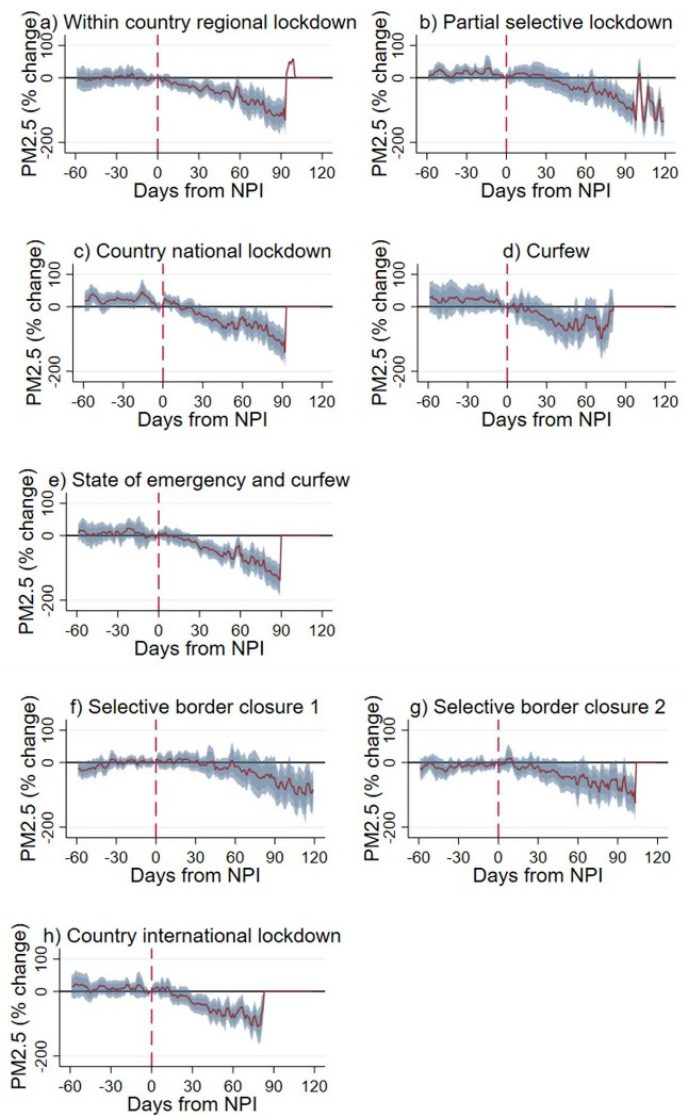

Figure 2. Non-pharmaceutical interventions reduced PM2.5 on the medium term similarly for inside measures (within country) and measures towards the outside (blocking the borders). More stringent measures tend to have larger effects (see panel (e) and (h)). 90\% and 99\% confidence intervals are shown in different shades of blue. The vertical dashed line shows the day when the measure was implemented and estimates are reported for as long as there is sufficient data to estimate them.

of lockdown, the pollution trend cannot be statistically distinguished from zero, suggesting the postlockdown pollution decline is a result of reduced human activity and not merely a continuation of a pre-existing pollution trend. Third, the pollution impact accumulates over time, showing the strongest reduction of around $100 \%$ at 90-120 days into lockdown. Crucially, countries that released their restrictions do not upward bias these results. Our release covariate ensures the release effect is subtracted away before identifying the lockdown effect.

There is a noticeable lag between lockdown implementations and air quality improvements. For inside measures, it takes over 1 month for PM2.5 declines to initiate $(p<0.05)$. For outside measures, it takes 2 months $(p<0.05)$. The delay is arguably because our categorization combines a continuum of behavioural changes. On one end, domestic lockdown measures include partial constraints like playground closures, and on the other end, full regional shutdowns.

Figure 2 separately shows the impact of various specific lockdown measures, always controlling for all other measures in place. For strict measures, e.g. a domestic regional lockdown (panel a), or a state of emergency with curfew (panel e), the timing of air quality improvements is remarkably coincident with the implementation date. For looser measures, e.g. a partial selective lockdown or a curfew (panels b and $\mathrm{d}^{14}$ ), the improvement in air pollution is only noticeable after a 1 month delay. Also, the first selective border closure (panel f) improves air quality only after about 45 days, while the second selective border closure already reduces air pollution after about 30 days (panel g). Countries which close their airports and international borders experience improvements in air quality after less than 30 days.

\subsection{Geographical heterogeneity}

Our main results describe the global average impact of lockdown on air quality. Of course, this masks potentially wide variation between different areas of the world. Providing country-level results is complicated for two reasons. First, country-specific political or socio-economic factors may change during the onset of lockdown, affecting both human activity and the environment. In the absence of such time-varying country controls (beyond our meteorological variables and COVID-19 deaths), we illustrate geographic heterogeneity with a pixel-level difference-in-difference map of PM2.5 (figure 3). Additionally, we average the urban pixel values in each country to document air quality changes during the first inside lockdown compared to before lockdown, relative to the same before-after periods in 2019 (table A1).

The second limitation is that our estimation strategy (equation (2)) relies on comparing daily air quality during lockdown to air quality on the day before lockdown. For a single country, this involves comparing two data points, making standard errors unobtainable. To address this, we categorize countries into geographic regions and then estimate equation (2).

Figure 3 shows that large areas in North and South America, Europe, Southern Africa, East Asia and the Pacific experience improvements in air quality relative to 2019. At the same time, however, some regions experience worse air quality during lockdown. Notably, PM2.5 increases in the American Midwest. This is consistent with [35], who find higher pollution in these areas due to a rollback in enforcement of environmental regulations during the pandemic. We also observe slight pollution increases in parts of the Sahara, Siberia, and Western China, mirroring the findings of [43] who produce a similar map using satellite AOD data. We are unable to conduct ground validation of pollution increases in these areas since

14 Although curfews can be considered strict, it is unlikely to starkly impact pollution because human activity is allowed to continue during the day until a specified evening hour. 


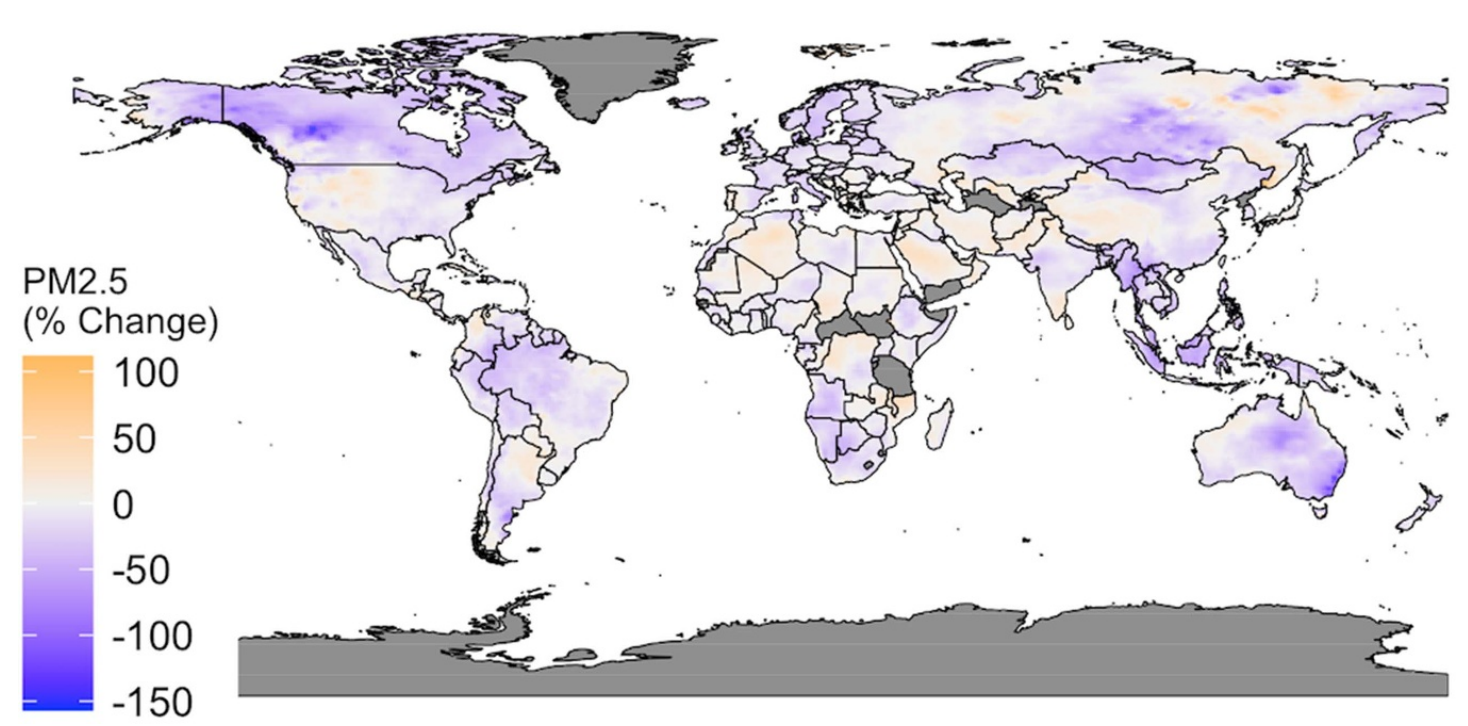

Figure 3. Global distribution of pandemic-induced air quality changes. Cell values describe the difference in mean log-PM2.5 before and after the first inside NPI measure relative to the same pre-post difference in 2019. The 'before' period extends to November of the previous year until the lockdown date. The 'after' period extends to 31 August.

such remote regions lack ground monitors and are largely absent from previous studies on COVID19 and pollution [31, 43]. Despite this limitation, these are largely unpopulated regions where relative changes in pollution are mostly driven by climate anomalies. Our formal estimates (equation (2)) are unaffected by this since we restrict the analysis to urban areas.

Table A1 in appendix summarizes differences in air pollution before and after lockdown by country. Again, there is substantial heterogeneity, with $65 \%$ of countries showing air quality improvements. Australian urban centres experience the biggest pollution reduction of $82 \%$. Note that differences the magnitude of pollution changes in table A1 may be driven by political factors, stringency of enforcement, or pollution spillovers between neighbouring countries. In our regional analysis, we account for many of these issues with the same fixed effects, covariates, and adjustments for spatial spillovers as in equation (2).

Figures 4 and 5 display the regional differences in the evolution of pollution in the short- and medium-run for inside measures and outside measures, respectively. Inside measures improve air quality in East Asia and the Pacific, South Asia, the Middle East, parts of North Africa, and in sub-Saharan Africa. Effects of outside measures are imprecisely estimated, and remain often insignificant. Regions with some significant improvements in air pollution include East Asia and the Pacific, South Asia, Latin America and the Caribbean, the Middle East and Northern Africa $^{15}$.

15 North America also experiences an improvement in air quality, but the number of observations is not sufficiently large to provide a statistical test for North-America.
Last but not least, figure 6 plots PM2.5 change against GDP changes during the COVID-19 pandemic, also taking into account the number of COVID-19 fatalities per capita (represented by the size of the dot) and the main sources of pollution (represented by the colour of the dot $)^{16}$. Several countries including Canada, Italy, Spain and the United States had substantial GDP losses as well as reductions in air pollution (all countries in the grey shaded area), yet there are also exceptions including Brazil, India, Japan and South Korea for which substantial GDP reductions were associated with increases in air pollution (all countries in the red shaded area). These differences may be explained by different sources of air pollution (as discussed in more depth in section 5 ).

\section{Discussion}

The wave of COVID-19 lockdowns provides an unprecedented opportunity, in an otherwise troubled time, to study the environmental consequences of reduced human activity. We document the short- and medium-term impact of this 'anthropause' (coined by [40]) on air quality by assembling the largest dataset of country-level NPIs and high-resolution PM2.5 concentrations across 176 countries. We find a global average reduction in PM2.5 concentrations of 35\%-45\% during COVID-19 lockdowns.

Our results mirror the direction of change in previous studies, but suggest larger air quality improvements. China was the first 'laboratory' for

16 Change is a 'difference-in-difference', i.e. the change between the first and second quarter in 2020 compared to the same change between the first and second quarter in 2019, both for PM2.5 and GDP. 


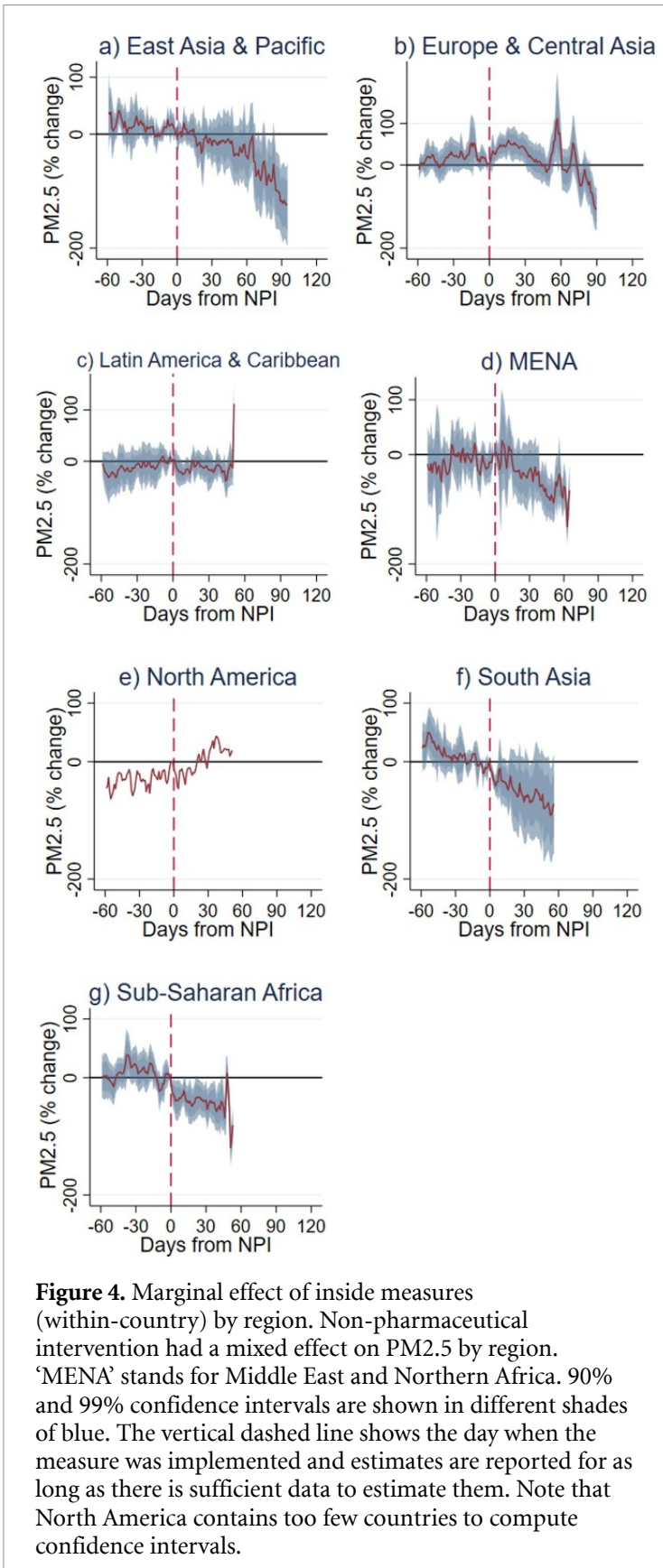

studying lockdown impacts, as it was the first country to impose this type of measure. A $30 \%-40 \%$ reduction in PM2.5 was found in four Chinese cities by [27] during 20 days of lockdown between January and February 2020. In contrast, [22] use a differencein-difference method in 300 cities and find a more muted reduction of $17 \%$. Our results are more comparable to global studies, that are limited in number. Venter et al [43] use ground monitor data in 34 countries and find an average PM2.5 reduction of $31 \%$, kicking in immediately after lockdown [31]. use the same monitor data as [43], but cover twice as many countries, and find a PM2.5 reduction half the size.

There are at least two explanations for our disparate results. First, our dataset is substantially richer
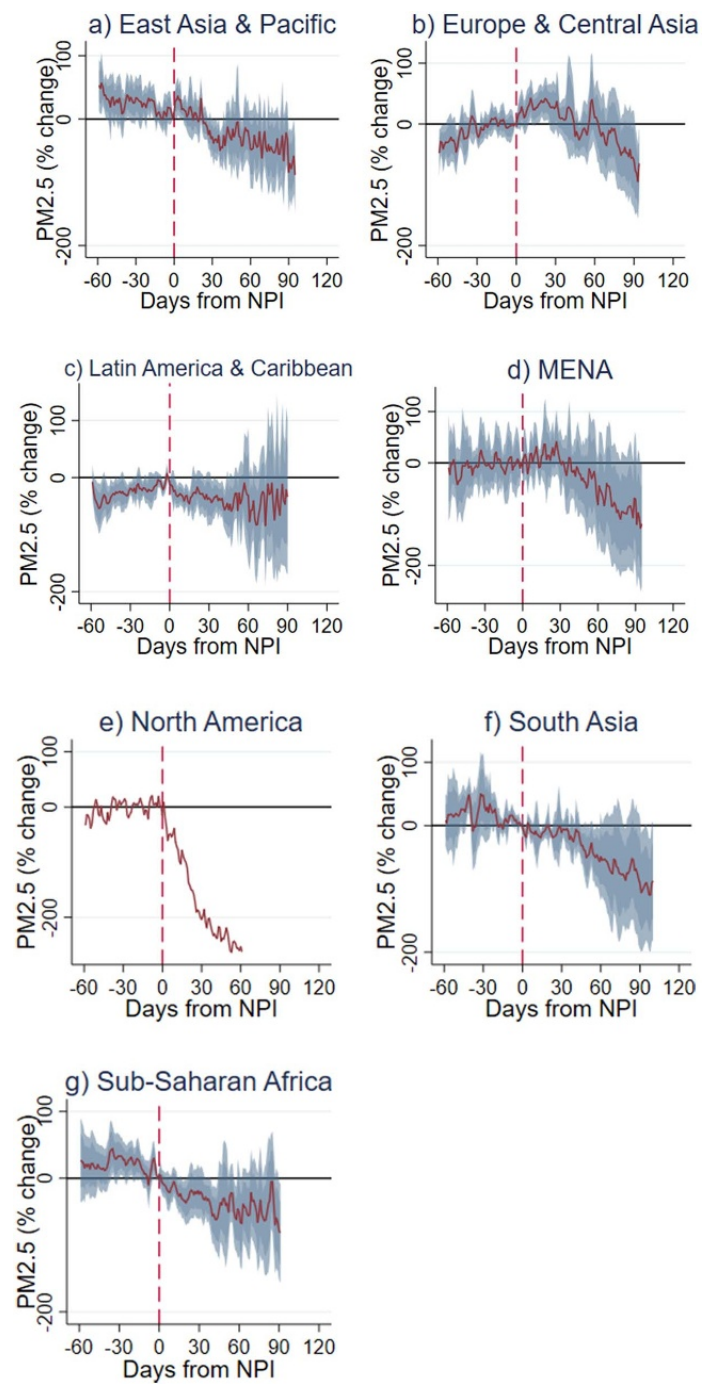

Figure 5. Marginal effect of borders closure (outside measures) by region. $90 \%$ and $99 \%$ confidence intervals are shown in different shades of blue. The vertical dashed line shows the day when the measure was implemented and estimates are reported for as long as there is sufficient data to estimate them. Note that North America contains too few countries to compute confidence intervals.

and spans locations experiencing large air quality reductions not covered by previous studies. For example, [31] cover 597 cities whereas we cover nearly 12000 . Africa, in particular, is virtually missing from both previous global studies, and South America is missing in [31]. But, as can be seen in figure 3 , these continents experienced sweeping air quality improvements during their lockdown. Second, shorter timelines in previous studies precluded the inclusion of release data, resulting in biased coefficients that bundle pollution reductions due to lockdown with pollution increases due to release, and appear smaller. In contrast, we separate out the effect of release measures and, as a result, find relatively larger air quality improvements.

Although most countries suffered from substantial economic losses during the first peak of the pandemic (figure 6) the results with respect to air 


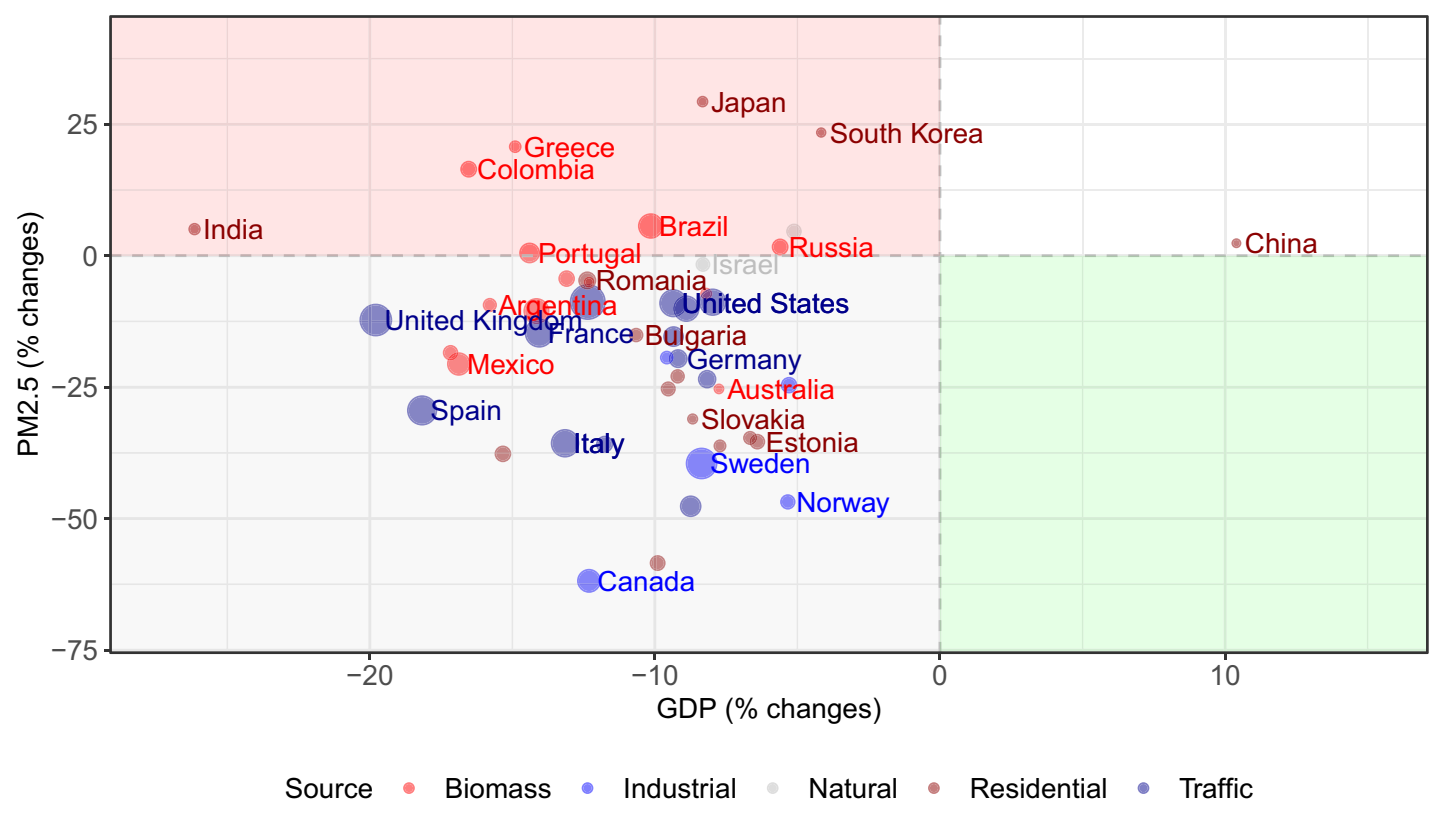

COVID-19 related deaths per million

Figure 6. Air quality changes in urban areas, GDP changes, sources of air pollution and COVID-19 related mortality. GDP and PM2.5 are measured as the difference in mean values between the first and second quarter in 2020 relative to the same difference in 2019. COVID-19 related deaths are the sum of deaths in the second quarter of 2020. The sources of air pollution are the sources responsible for the largest impact of PM2.5 on mortality in 2010 [29]. The GDP data are from the OECD while Covid deaths data are from the European Centre for Disease Prevention and Control (see section 2.3).

pollution are heterogeneous. Consistent with a positive association between air pollution and economic activity, many countries suffered substantial GDP losses as well as reductions in air pollution. However, some countries experienced substantial GDP reductions with increases in pollution-so their lockdowns slowed down their economies but did not improve the quality of their air. These differences may be explained by different sources of air pollution. While transportation and industry plays an important role for air pollution in densely populated parts of Europe and North America, air pollution in Latin America and Asia is dominated by biomass burning, agriculture and residential energy use (e.g. Extended Data figure 1 of [29]). The different sources of air pollution may respond differently to COVID-19 lockdowns. For example, lockdown measures are likely to increase residential energy use (mainly for heating) while they may reduce pollution from transportation including commuting. Biomass burning is largely related to agriculture which was generally little affected by lockdowns. However, figure 6 also suggests that win-win situations are unlikely to occur as there is no country with increases in GDP and reductions in air pollution (green shaded quadrant). The economic growth of China during the second quarter of 2020 may be attributed to recovery from the earlier COVID-19 outbreak in China.

Figure 6 therefore suggests that a reduction in economic activity or mobility might not necessarily save the environment. In fact, economic downturns can increase air pollution if they lead to a shift of economic activities that are more harmful to the environment. This non-linear relationship between economic growth and environmental quality due to changes in the composition of economic activity is discussed in the large literature on economic growth and the environment (see e.g. [20], [4] or [8] for overviews). We contribute to this literature by providing evidence for similar relationships in the short and the medium-run.

Prohibiting economic activity does improve air quality in the majority of countries around the world, but the price is very high, and in some countries air quality does not improve even though the economy comes to a halt. In turn, economic growth may not necessarily lead to environmental degradation. A shift from more polluting activities to less polluting activities during the growth process such as the shift from resource based activities associated with biomass burning to manufacturing, and later services, can simultaneously increase economic prosperity as well as air quality. However, the necessary changes to improve air quality may differ across economies. A policy to discourage commuting may improve air quality in one region while the same policy may have the reverse effect in a region for which increasing decentralized economic activities is associated with elevated pollution levels. These findings underline the importance of market-based environmental instruments such as Pigouvian taxes or cap and trade systems to reduce pollution at the lowest possible costs. 
However, they also stress the importance of including all economic activities in these regulations as substitution between activities in response to regulation may worsen the situation. Although the pandemic has caused substantial losses in economic prosperity and human lives it has also improved the environment. The large heterogeneity in the relation between economic activity and air quality suggests that improving the environment may not require these sacrifices.

\section{Data availability statement}

The data that support the findings of this study are available upon reasonable request from the authors.

\section{Acknowledgments}

Rafael Lalive acknowledges funding from the National Center of Competence in Research LIVES. 
Appendix

Table A1. Change in PM2.5 during lockdown relative to 2019.

\begin{tabular}{|c|c|c|c|c|c|}
\hline Country & PM2.5 (Pct.) & Country & PM2.5 (Pct.) & Country & PM2.5 (Pct.) \\
\hline Afghanistan & -2.87 & Gambia & 17.45 & Nicaragua & 7.32 \\
\hline Albania & -10.34 & Georgia & -13.94 & Niger & -10.23 \\
\hline Algeria & -19.97 & Germany & -13.09 & Nigeria & -14.51 \\
\hline Andorra & -35.64 & Ghana & -21.85 & North Macedonia & 9.5 \\
\hline Angola & -18.36 & Greece & 15.87 & Norway & -35.13 \\
\hline Antigua and Barbuda & -23.38 & Guatemala & 25.28 & Oman & 4.92 \\
\hline Argentina & 2.62 & Guinea & -2.7 & Pakistan & 9.4 \\
\hline Armenia & 5.48 & Guinea-Bissau & 21.68 & Panama & -0.11 \\
\hline Australia & -82.11 & Guyana & -6.6 & Papua New Guinea & 14.18 \\
\hline Austria & -1.74 & Haiti & 4.64 & Paraguay & 14.53 \\
\hline Azerbaijan & 23.22 & Honduras & 11.41 & Peru & -7.51 \\
\hline Bahamas & -7.65 & Hungary & -8.61 & Philippines & -31.16 \\
\hline Bahrain & 8.04 & Iceland & -10.09 & Poland & -7.52 \\
\hline Bangladesh & -22.71 & India & -6.92 & Portugal & 25.79 \\
\hline Barbados & -7.78 & Indonesia & -31.86 & Qatar & 6.38 \\
\hline Belarus & -26.2 & Iran & 8.62 & Romania & 5.87 \\
\hline Belgium & -17.78 & Iraq & 1.71 & Russia & -5.78 \\
\hline Benin & -17.61 & Ireland & -12.25 & Rwanda & -16.15 \\
\hline Bolivia & -27.31 & Israel & 7.38 & Saint Kitts and Nevis & -21.99 \\
\hline Bosnia and Herzegovina & 18.27 & Italy & -32.3 & Saudi Arabia & 3.6 \\
\hline Botswana & -18.93 & Ivory Coast & -7.85 & Senegal & 14.1 \\
\hline Brazil & -3.63 & Jamaica & -12.38 & Serbia & 10.39 \\
\hline Brunei & -40.56 & Japan & 2.98 & Sierra Leone & -9.69 \\
\hline Bulgaria & 10.18 & Jordan & -0.14 & Singapore & -59.91 \\
\hline Burkina Faso & -6.56 & Kazakhstan & -2.12 & Slovakia & -16.59 \\
\hline Burma & -32.12 & Kenya & -1.56 & Slovenia & -6.96 \\
\hline Cambodia & -27.8 & Kosovo & 15.38 & Somalia & -0.19 \\
\hline Cameroon & -2.57 & Kuwait & -6.36 & South Africa & -8.91 \\
\hline Canada & -29.54 & Kyrgyzstan & 0.49 & South Korea & -14.28 \\
\hline Chad & 10.74 & Laos & 1.47 & Spain & -12.64 \\
\hline Chile & -20.98 & Latvia & -30.22 & Sri Lanka & 8.23 \\
\hline China & -5.81 & Lebanon & 14.04 & Sudan & 6.19 \\
\hline Colombia & 6.35 & Liberia & -13.14 & Suriname & -15.24 \\
\hline Congo (Brazzaville) & -2.38 & Libya & 6.76 & Sweden & -40.57 \\
\hline Congo (Kinshasa) & -2.18 & Lithuania & -19.75 & Switzerland & -14.24 \\
\hline Costa Rica & 6.94 & Luxembourg & -14.9 & Syria & 3.91 \\
\hline Croatia & 5.49 & Madagascar & -11.62 & Taiwan & -8.18 \\
\hline Cuba & 16.9 & Malawi & 21.99 & Thailand & -19.16 \\
\hline Cyprus & 11.15 & Malaysia & -47.19 & Togo & -17.56 \\
\hline Czech Republic & -2.58 & Mali & 0.76 & Trinidad and Tobago & -31.04 \\
\hline Denmark & -44.35 & Mauritania & -8.69 & Tunisia & -16.91 \\
\hline Djibouti & -19.13 & Mauritius & -30.27 & Turkey & 0.38 \\
\hline Dominican Republic & -14.36 & Mexico & -5.08 & Uganda & -8.71 \\
\hline Ecuador & 1.05 & Moldova & -8.89 & Ukraine & -1.7 \\
\hline Egypt & 2.99 & Mongolia & -44.02 & United Arab Emirates & 13.36 \\
\hline El Salvador & 15.37 & Montenegro & -1.03 & United Kingdom & -18.75 \\
\hline
\end{tabular}


Table A1. (Continued.)

\begin{tabular}{llllll}
\hline Country & PM2.5 (Pct.) & Country & PM2.5 (Pct.) & Country & PM2.5 (Pct.) \\
\hline Eritrea & -18.79 & Morocco & 6.86 & Uruguay & 6.71 \\
Estonia & -37.77 & Mozambique & 8.94 & US & 8.4 \\
Ethiopia & -32.37 & Namibia & -0.16 & Uzbekistan & 12.06 \\
Finland & -24.02 & Nepal & -22.64 & Venezuela & -7.66 \\
France & -17.52 & Netherlands & -20.82 & Vietnam & -21.27 \\
Gabon & 1 & New Zealand & -12.7 & Zambia & 5.22 \\
& & & & Zimbabwe & -8.67
\end{tabular}

Note: Values represent the percentage difference-in-difference of PM2.5. This is calculated as follows. First, we calculate mean PM2.5 across all urban areas from the first lockdown until 31 August 2020 (post-period). We then do the same from 1 November 2019 until the lockdown (pre-period). Second, we do the same calculation in the previous year adjusting for differences in calendar days. For example, if lockdown was imposed on 1 March 2020 (a Sunday), then we set the first Sunday of March in 2019 as the start of the 2019 post-period (instead of the same calendar date). Lastly, we take the natural log of each pre and post mean and compute the difference in difference.

\section{ORCID iDs}

Rafael Lalive (1) https://orcid.org/ 0000-0003-1938$692 X$

Raahil Madhok (1) https://orcid.org/0000-00034434-2785

Frederik Noack (1) https://orcid.org/0000-00025747-4368

Dominic Rohner (ㄱ) https://orcid.org/0000-00032660-4550

Tommaso Sonno (1) https://orcid.org/0000-00025697-9647

\section{References}

[1] Al-Abadleh H A, Lysy M, Neil L, Patel P, Mohammed W and Khalaf Y 2020 Statistical quantification of COVID-19 lockdown effect on air quality from ground-based measurements in Ontario, Canada (ChemRxiv)

[2] Bonardi J-P, Bris A, Brülhart M, Danthine J-P, Jondeau E, Rohner D and Thoenig M 2020 The case for reopening economies by sector Harv. Bus. Rev.

[3] Bonardi J-P, Gallea Q, Kalanoski D and Lalive R 2020 Fast and local: lockdown policies affect the spread and severity of COVID-19 Covid Econ. 23 325-51

[4] Brock W A and Taylor M S 2005 Economic growth and the environment: a review of theory and empirics Handbook Econ. Growth 1 1749-821

[5] Brodeur A, Cook N and Wright T 2020 On the effects of COVID-19 safer-at-home policies on social distancing, car crashes and pollution IZA Discussion Paper No. 13255

[6] Buchard V et al 2017 The MERRA-2 aerosol reanalysis, 1980 onward. Part II: evaluation and case studies J. Clim. $306851-72$

[7] Buchard V, da Silva A, Randles C, Colarco P, Ferrare R, Hair J, Hostetler C, Tackett J and Winker D 2016 Evaluation of the surface PM2.5 in version 1 of the NASA MERRA aerosol reanalysis over the United States Atmos. Environ. 125 100-11

[8] Carson R T 2010 The environmental Kuznets curve: seeking empirical regularity and theoretical structure Rev. Environ. Econ. Policy 4 3-23

[9] Chaloulakou A, Kassomenos P, Spyrellis N, Demokritou P and Koutrakis P 2003 Measurements of PM10 and PM2. 5 particle concentrations in Athens, Greece Atmos. Environ. 37 649-60

[10] Chang H-H, Meyerhoefer C and Yang F-A 2020 COVID-19 prevention and air pollution in the absence of a lockdown National Bureau of Economic Research WP No. 27604
[11] Chin M et al 2002 Tropospheric aerosol optical thickness from the GOCART model and comparisons with satellite and sun photometer measurements J. Atmos. Sci. 59 461-83

[12] Cicala S, Holland S P, Mansur E T, Muller N Z and Yates A J 2020 Expected health effects of reduced air pollution from covid-19 social distancing Technical Report National Bureau of Economic Research

[13] Csavina J, Field J, Félix O, Corral-Avitia A Y, Sáez A E and Betterton E A 2014 Effect of wind speed and relative humidity on atmospheric dust concentrations in semi-arid climates Sci. Total Environ. 487 82-90

[14] Dang H-A and Trinh T-A 2020 The beneficial impacts of COVID-19 lockdowns on air pollution: evidence from Vietnam IZA Discussion Paper No. 13651

[15] Dang H-A and Trinh T-A 2020 Does the COVID-19 pandemic improve global air quality? New cross-national evidence on its unintended consequences IZA Discussion Paper No. 13480

[16] Dong E, Du H and Gardner L 2020 An interactive web-based dashboard to track COVID-19 in real time Lancet Infect. Dis. 20 533-4

[17] Forster P M et al 2020 Current and future global climate impacts resulting from COVID-19 Nat. Clim. Change 10 913-9

[18] Gelaro Ret al 2017 The modern-era retrospective analysis for research and applications, version 2 (MERRA-2) J. Clim. 30 5419-54

[19] Grainger C and Schreiber A 2019 Discrimination in ambient air pollution monitoring? AEA Papers and Proc. vol 109 pp 277-82

[20] Grossman G M and Krueger A B 1995 Economic growth and the environment Q. J. Econ. 110 353-77

[21] Gupta P, Christopher S A, Wang J, Gehrig R, Lee Y and Kumar N 2006 Satellite remote sensing of particulate matter and air quality assessment over global cities Atmos. Environ. 40 5880-92

[22] He G, Pan Y and Tanaka T 2020 The short-term impacts of COVID-19 lockdown on urban air pollution in China Nat. Sustain. 3 1005-11

[23] He L, Lin A, Chen X, Zhou H, Zhou Z and He P 2019 Assessment of MERRA-2 surface PM2.5 over the Yangtze River Basin: ground-based verification, spatiotemporal distribution and meteorological dependence Remote Sens. 11460

[24] Hsiang S et al 2020 The effect of large-scale anti-contagion policies on the COVID-19 pandemic Nature 584 262-7

[25] Huffman G J, Bolvin D T, Braithwaite D, Hsu K, Joyce R, Xie P and Yoo S-H 2015 Nasa global precipitation measurement (GPM) integrated multi-satellite retrievals for GPM (IMERG) Algorithm Theoretical Basis Document (ATBD) Version 4:26 
[26] Landrigan P J et al 2018 The lancet commission on pollution and health Lancet $391462-512$

[27] Le T, Wang Y, Liu L, Yang J, Yung Y L, Li G and Seinfeld J H 2020 Unexpected air pollution with marked emission reductions during the COVID-19 outbreak in China Science $369702-6$

[28] Le Quéré C et al 2020 Temporary reduction in daily global $\mathrm{CO}_{2}$ emissions during the COVID-19 forced confinement Nat. Clim. Change 10 647-53

[29] Lelieveld J, Evans J S, Fnais M, Giannadaki D and Pozzer A 2015 The contribution of outdoor air pollution sources to premature mortality on a global scale Nature 525 367-71

[30] Li J, Carlson B E and Lacis A A 2015 How well do satellite AOD observations represent the spatial and temporal variability of PM2.5 concentration for the united states? Atmos. Environ. 102 260-73

[31] Liu F, Wang M and Zheng M 2021 Effects of COVID-19 lockdown on global air quality and health Sci. Total Environ. 755142533

[32] Luan T, Guo X, Guo L and Zhang T 2018 Quantifying the relationship between PM2.5 concentration, visibility and planetary boundary layer height for long-lasting haze and fog-haze mixed events in Beijing Atmos. Chem. Phys. 18203

[33] Newbold S C, Finnoff D, Thunström L, Ashworth M and Shogren J F 2020 Effects of physical distancing to control COVID-19 on public health, the economy and the environment Environ. Resour. Econ. 76 705-29

[34] Paciorek C J and Liu Y 2009 Limitations of remotely sensed aerosol as a spatial proxy for fine particulate matter Environ. Health Perspect. 117 904-9

[35] Persico C and Johnson K R 2020 Deregulation in a time of pandemic: does pollution increase Coronavirus cases or deaths? IZA Discussion Papers 13231, Institute of Labor Economics (IZA) (available at: https://ideas.repec.org/p/iza/ izadps/dp13231.html)
[36] Provençal S, Buchard V, Silva A, Leduc R, and Barrette N 2016 Evaluation of PM2.5 surface concentration simulated by version 1 of the NASA's MERRA aerosol reanalysis over Israel and Taiwan. Aerosol Air Qual. Res. 17

[37] Provençal S, Buchard V, da Silva A M, Leduc R and Barrette N 2017 Evaluation of PM surface concentrations simulated by version 1 of NASA's MERRA aerosol reanalysis over Europe Atmos. Pollut. Res. $8374-82$

[38] Rienecker M M et al 2008 The GEOS-5 data assimilation system: documentation of versions 5.0. 1, 5.1. 0 , and 5.2. 0

[39] Ropkins K and Tate J 2020 Early observations on the impact of the COVID-19 lockdown on air quality trends across the UK Sci. Total Environ. 754142374

[40] Rutz C et al 2020 COVID-19 lockdown allows researchers to quantify the effects of human activity on wildlife Nat. Ecol. Evol. 4 1156-9

[41] Schneider A, Friedl M A and Potere D 2009 A new map of global urban extent from MODIS satellite data Environ. Res. Lett. 4044003

[42] Tyagi P, Braun D, Sabath B, Henneman L and Dominici F 2020 Short-term change in air pollution following the COVID-19 state of emergency: a national analysis for the United States (medRxiv)

[43] Venter Z S, Aunan K, Chowdhury S and Lelieveld J 2020 COVID-19 lockdowns cause global air pollution declines Proc. Natl Acad. Sci. 117 18984-90

[44] Wang Z, Uno I, Yumimoto K, Itahashi S, Chen X, Yang W and Wang Z 2021 Impacts of COVID-19 lockdown, spring festival and meteorology on the $\mathrm{NO}_{2}$ variations in early 2020 over China based on in-situ observations, satellite retrievals and model simulations Atmos. Environ. 244117972

[45] Yan X, Zang Z, Zhao C and Husi L 2021 Understanding global changes in fine-mode aerosols during 2008-2017 using statistical methods and deep learning approach Environ. Int. 149106392 